\title{
VELOCITY FIELDS OF BLOOD FLOW IN MICROCHANNELS USING A CONFOCAL MICRO-PIV SYSTEM
}

\author{
RUI LIMA ${ }^{1,2}$, TAKUJI ISHIKAWA ${ }^{1}$, SHUJI TANAKA ${ }^{3}$, MOTOHIRO TAKEDA ${ }^{1,4}$, \\ KEN-ICHI TSUBOTA ${ }^{1}$, SHIGEO WADA ${ }^{5}$, TAKAMI YAMAGUCHI ${ }^{1}$
}

1) Dept. Bioeng. \& Robotics, Grad. Sch. Eng., Tohoku Univ., 6-6-01 Aoba, 980-8579

Sendai, Japan.

2) Dept. Mechanical Tech., ESTiG, Braganca Polyt., C. Sta. Apolonia, 5301-857

Braganca, Portugal.

3) Dept. Nanomechanics, Grad. Sch. Eng., Tohoku Univ., 6-6-01 Aoba, 980-8579 Sendai, Japan.

4) Div. Surgical Oncology, Grad. Sch. Medicine, Tohoku Univ., 2-1 Seiryo-machi, Aobaku, 980-8575 Sendai, Japan.

5) Dept. Mechanical Science and Bioeng., Grad. Sch. Eng., Osaka Univ., Toyonaka, 5608531 Osaka, Japan.

\begin{abstract}
The in vitro experimental investigations provide an excellent approach to understand complex blood flow phenomena involved at a microscopic level. This paper emphasizes an emerging experimental technique capable to quantify the flow patterns inside microchannels with high spatial and temporal resolution. This technique, known as confocal micro-PIV, consists of a spinning disk confocal microscope, high speed camera and a diode-pumped solid state (DPSS) laser. Velocity profiles of pure water (PW), physiological saline (PS) and in vitro blood were measured in a $100 \mu \mathrm{m}$ glass square and rectangular polydimethysiloxane (PDMS) microchannel. The good agreement obtained between measured and estimated results suggests that this system is a very promising technique to obtain detail information about micro-scale effects in microchannels by using both homogeneous and non-homogeneous fluids such as blood flow.
\end{abstract}

\section{Introduction}

Detailed knowledge on flow velocity profiles of blood flow in microchannels is essential to provide a better understanding on the blood rheological properties and disorders in microvessels. Despite the high amount of research in microcirculation, there is not yet any detailed experimental information about flow velocity profiles, RBCs deformability and aggregation in microvessels. This lack of knowledge is mainly due to the absence of adequate techniques to measure and quantitatively evaluate fluid mechanical effects at a microscopic level [1, 2].

During the years the most research work in this area has focused in experimental studies using techniques such as laser Doppler anemometry (LDA) 
or conventional particle image velocimetry (PIV). However, due to limitations of those techniques to study effects at a micro-scale level, Meinhart and his colleagues [3] have proposed a measurement technique that combines the PIV system with an inverted epi-fluorescent microscope, which increases the resolution of the conventional PIV systems [3, 4]. More recently, considerable progress in the development of confocal microscopy and consequent advantages of this microscope over the conventional microscopes have led to a new technique known as confocal micro-PIV [5-9]. This technique combines the conventional PIV system with a spinning disk confocal microscope (SDCM). Due to its outstanding spatial filtering technique together with the multiple point light illumination system, this kind of microscope has the ability to obtain infocus images with optical thickness less than $1 \mu \mathrm{m}$, task extremely difficult to be achieved by using a conventional microscope. As a result, by combining SDCM with the conventional PIV system it is possible to achieve a PIV system with not only extremely high spatial resolution but also with capability to generate 3D velocity profiles.

The main purpose of this paper is to investigate the ability of the confocal micro-PIV system to measure velocity fields of both homogeneous and nonhomogeneous fluids in two different kinds of microchannels, i. e., $100 \mu \mathrm{m}$ glass square and rectangular (300 $\mu \mathrm{m}$ wide, $45 \mu \mathrm{m}$ deep) PDMS microchannel.

\section{Materials and methods}

\subsection{Working fluids and microchannels}

Four working fluids were used in this study: pure water (PW), physiological saline (PS) and PS containing about $17 \%(17 \mathrm{Hct})$ and $20 \%(20 \mathrm{Hct})$ of human red blood cells (RBCs). All fluids were seeded with 0.1 to $0.15 \%$ (by volume) 1 $\mu \mathrm{m}$-diameter red fluorescent solid polymer microspheres (R0100; Duke Scientific, USA). The blood was collected from a healthy adult volunteer, where ethylenediaminetetraacetic acid (EDTA) was added to prevent coagulation. The RBCs where separated from the bulk blood by centrifugation and aspiration of the plasma and buffy coat and then washed twice with PS. The washed RBCs where diluted with PS to make up the required RBCs concentration by volume. All blood samples were stored hermetical at $4^{\circ} \mathrm{C}$ until the experiment was performed at room temperature $\left(25\right.$ to $\left.27^{\circ} \mathrm{C}\right)$.

Two microchannels were used in this study. The first was $100 \mu \mathrm{m} \times 100 \mu \mathrm{m}$ borosilicate glass square microchannel fabricated by Vitrocom. The square microchannel was mounted on a slide glass with thickness of approximately 120 $\mu \mathrm{m}$, which was immersed in glycerine in order to minimize some possible refraction from the walls [9]. A second microchannel was a PDMS rectangular 
microchannel $(300 \mu \mathrm{m}$ wide, $45 \mu \mathrm{m}$ deep) fabricated by a soft lithographic technique at the Esashi, Ono and Tanaka Laboratory, Department of Nanomechanics, Tohoku University [10].

\subsection{Confocal micro-PIV experimental set-up}

The confocal micro-PIV system used in our experiment consists of an inverted microscope (IX71, Olympus, Japan) combined with a confocal scanning unit (CSU22, Yokogawa, Japan) and a diode-pumped solid state (DPSS) laser (Laser Quantum Ltd, England) with an excitation wavelength of $532 \mathrm{~nm}$. Moreover, a high-speed camera (Phantom v7.1, U.S.A.) was connected into the outlet port of the CSU22. The microchannels were placed on the stage of the inverted microscope where the flow rate of the working fluids was kept constant by means of a syringe pump (KD Scientific Inc., U.S.A.). For the square microchannel the flow rate was $0.15 \mu \mathrm{l} / \mathrm{min}(\operatorname{Re}=0.014)$, whereas for the PDMS rectangular microchannel the flow rate was $0.22 \mu 1 / \min (\operatorname{Re}=0.021)$.

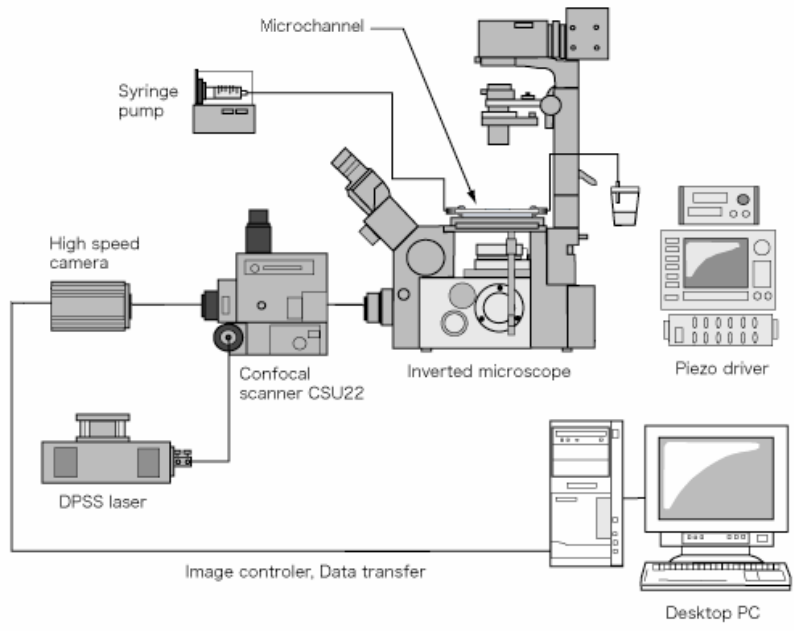

Fig. 1. Confocal micro-PIV experimental set-up.

The microscopic observations and the capturing of the confocal PIV images were performed in the middle of the microchannels. By using a RT3D software it was possible to collect a series of xy confocal images at different $\mathrm{z}$ positions. The images were captured with a resolution of $640 \times 480$ pixels, 12-bit grayscale, at a rate of $200 \mathrm{frames} / \mathrm{s}$ with an exposure time of $4995 \mathrm{~ms}$. The recorded images were transferred to the computer and then evaluated by the PivView version 2.3 (PivTec) using a cross-correlation method [11]. As a result it was possible to obtain the velocity vector fields at the interrogation area of interest. Deatailed 
information about the experimental set-up, used in the present study, has already been described previously [9].

\section{Results and discussion}

\subsection{Comparison between Experimental and Analytical Results}

By using the optical sectioning ability of the confocal system it was possible to obtain series of optical sectioned images along $\mathrm{z}$ axis. Figures 2 and 3 shows a comparison between analytical solutions [12] and average fluid velocities of 100 PIV image pairs at several optical sectioned images for both microchannels. Generally, the experimental results from Figures 2 and 3 show good agreement with the analytical solution, especially the results from the middle plane where we found errors less than 5\%. As one moves out of the middle plane the deviations start to increase and at locations close to the wall the errors can be slightly larger than $10 \%$. We believe that these latter errors are mainly attributable to "second-order effects" such as the surface roughness of the wall and background noise generated from particles adhered into the wall.

The results from Figure 3 also show that the velocity patterns were approximately parabolic which is characteristic of laminar flow in a square microchannel. In contrast, the velocity profiles from the PDMS microchannel (see Figure 4) were markedly flat in the central region, which is characteristic of laminar flow in a rectangular microchannel with low aspect ratio $(\mathrm{h} / \mathrm{w}=0.15)$.

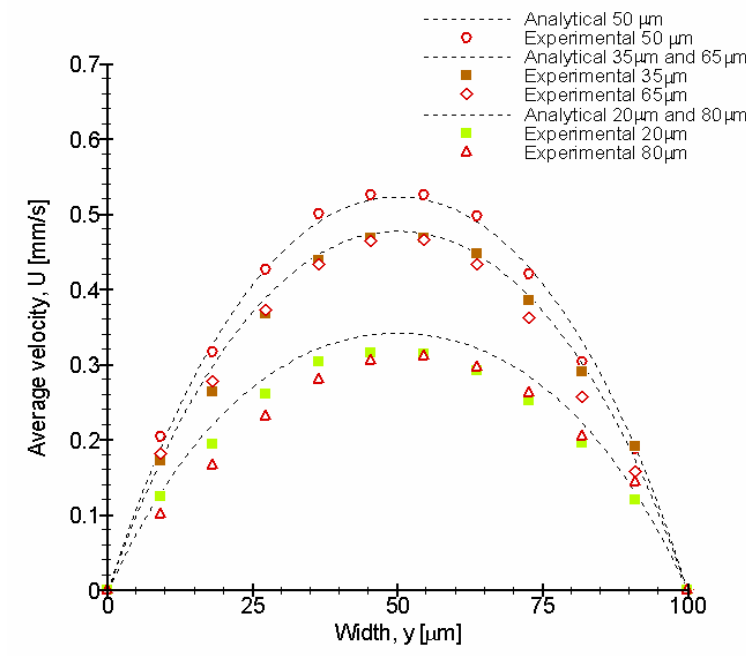

Fig. 2. Comparison between experimental data and analytical solutions at several optical sectioned images in $100 \mu \mathrm{m}$ square microchannel. The working fluid is PW. 


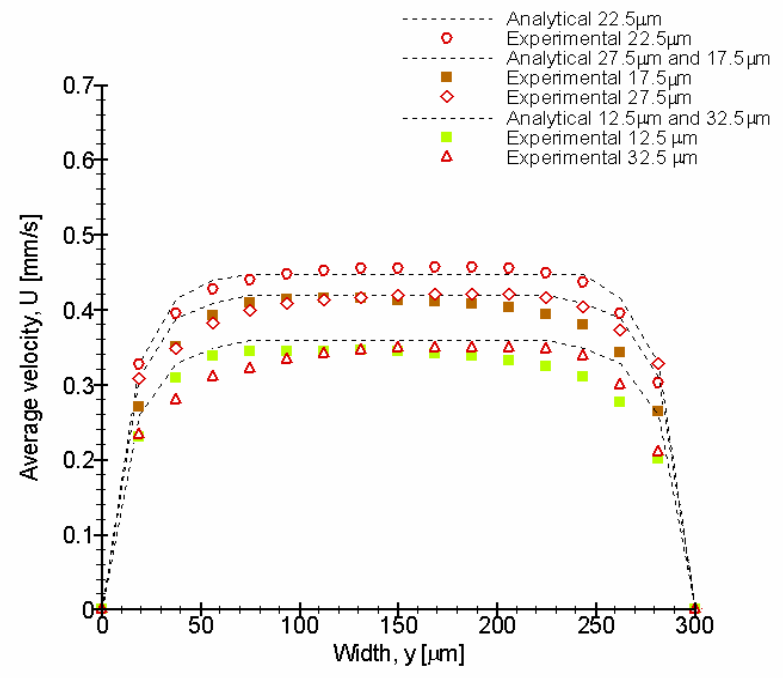

Fig. 3. Comparison between experimental data and analytical solutions at several optical sectioned images in a rectangular PDMS microchannel. The working fluid is PS.

\subsection{Velocity fields of in vitro blood flow}

Besides the employment of PW and PS, in this study PS containing around $17 \%$ and $20 \%$ of suspended blood cells was also used in order to evaluate the potentialities of the confocal micro-PIV system to investigate the flow behaviour of in vitro blood in both microchannels.

Figure 4 and 5 show the ability of the system to obtain velocity vector fields of in vitro blood at different xy planes. However, for the case of the square microchannel it was not possible to obtain reliable results along the full depth of the microchannel, especially in the upper part. One of the reasons for this limitation is the thickness of the wall and slide glass which imply a much high working distance to obtain the velocity fields. Another reason is the high concentration of RBCs in the middle region of the square microchannel, which decreases the intensity of the emitted light from the flowing fluorescent particles within the plasma flow, especially from the upper part of the microchannel. Conversely, for the case of the PDMS microchannel it was possible to record images along the full depth, in order to be processed by the PIV software. In this latter microchannel, due mainly to its geometry, we did not visualized any plasma layer, but rather a homogenous distribution of the RBCs which could contribute to the improvement on the images quality recorded by the high speed camera. 


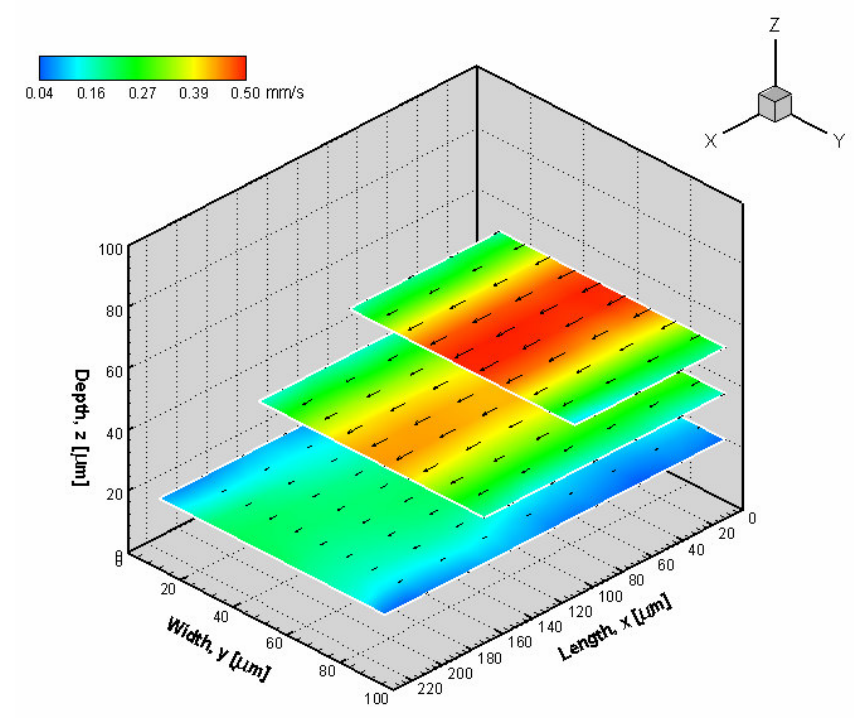

Fig. 4. Velocity vector fields from several optical sectioned images along $\mathrm{z}$ axis, in a $100 \mu \mathrm{m}$ glass square microchannel. The working fluid is in vitro blood with $17 \%$ Hct.

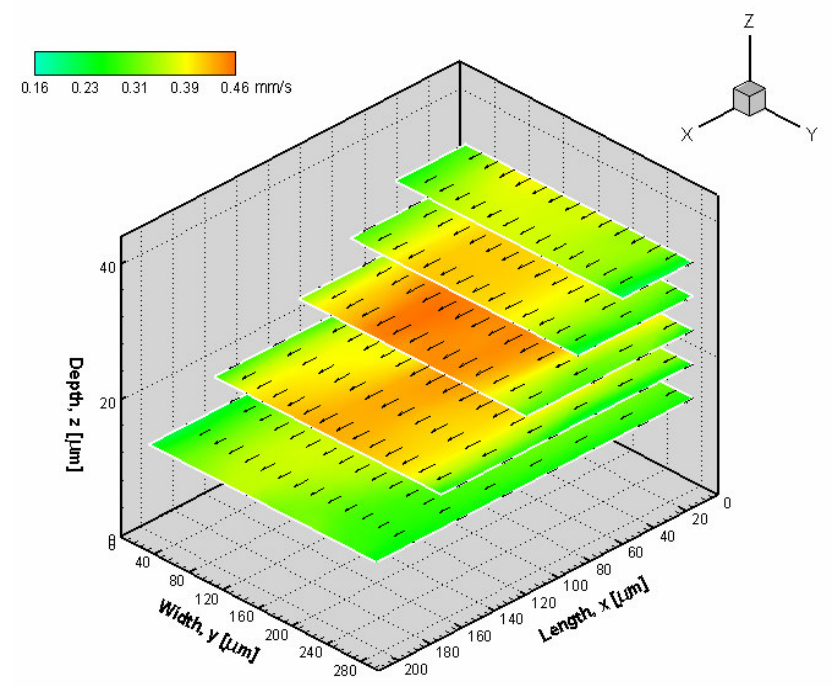

Fig. 5. Velocity vector fields from several optical sectioned images along $\mathrm{z}$ axis, in a rectangular PDMS microchannel. The working fluid is in vitro blood with $20 \%$ Hct. 


\subsection{Comparison between glass and PDMS microchannels}

Although it is possible to manufacture glass microchannels with circular or rectangular cross sections this kind of microchannels are limited to only straight geometries. Thus, it is crucial to use a microfabrication technique able to produce microfluidic devices with complex geometries similar to human blood arterioles and capillaries networks. The PDMS microchannel used in this study have shown a number of very useful properties to study in vitro blood flow, such as good optical transparency, easy reversible sealing to glass, good mechanical rigidity, chemical inertness, low toxicity, low cost and ability to have very complex geometries. Furthermore, due to the small working distance (less 100 $\mu \mathrm{m})$ required to obtain confocal images, the PDMS microchannel have shown to be extremely suitable to study several phenomena in microcirculation by combining it with our confocal micro-PIV system.

\section{Conclusions}

We applied a confocal micro-PIV system to measure PW, PS and in vitro blood flowing in two kinds of micorchannels, i. e., glass square and rectangular PDMS microchannel. The measured velocity profiles of PW and PS agree well with established analytical solution. Moreover, the measurements of in vitro blood $(17 \%$ and $20 \% \mathrm{Hct}$ ) have demonstrated the ability of this system to obtain confocal images with just the fluorescent particles within the plasma flow. As a result, the confocal micro-PIV system has demonstrated potential to obtain accurate detail information about micro-scale effects in both microchannels.

This study also demonstrated that the combination of confocal micro-PIV systems with PDMS microchannels would play an important role in the assessment of several phenomena in microcirculation.

\section{Acknowledgments}

This study was supported in part by the following grants: 21 st Century COE Program for Future Medical Engineering based on Bio-nanotechnology, International Doctoral Program in Engineering from the Ministry of Education, Culture, Sports, Science and Technology of Japan (MEXT), "Revolutionary Simulation Software (RSS21)" next-generation IT program of MEXT; Grantsin-Aid for Scientific Research from MEXT and JSPS Scientific Research in Priority Areas (768) "Biomechanics at Micro- and Nanoscale Levels," Scientific Research (A) No.16200031 "Mechanism of the formation, destruction, and movement of thrombi responsible for ischemia of vital organs." The authors also 
thank all members of Esashi, Ono and Tanaka Lab. for their assistance in fabricating the PDMS microchannel.

\section{References}

1. J. Lee, 2000 Distinguished Lecture: Biomechanics of the Microcirculation, An Integrative and Therapeutic Perspective, Annals of Biomedical Eng. 28, 1 (1998).

2. G. Mchedlishvili, and N. Maeda, Blood Flow Structure Related to Red Cell Flow: A Determination of Blood Fluidity in Narrow Microvessels, Japanese Journal of Physiology 51, 19 (2001).

3. C. Meinhart, S. Wereley and J. Santiago, PIV Measurements of a Microchannel Flow, Experiments in Fluids, 27, 414 (1999).

4. N. Nguyen and S. Wereley, Fundamentals and applications of microfluidics. Norwood MA: Artech House Inc, (2002).

5. T. Tanaani, S. Otsuki, N. Tomosada, Y. Kosugi, M. Shimizu and H. Ishida High-speed 1-frame/ms scanning confocal microscope with a microlens and Nipkow disks, Applied Optics, 41, 22, 4704 (2002)

6. J. Park, C. Choi and K. Kihm, Optically sliced micro-PIV using confocal laser scanning microscopy, Experiments in Fluids, 37, 105 (2004).

7. H. Kinoshita, M. Oshima, S. Kaneda and T. Fujii, Confocal micro-PIV measurement of internal flow in a moving droplet. Proc. $9^{\text {th }}$ ICMSCLS Boston, Massachusetts, 629 (2005).

8. R. Lima, S. Wada, K. Tsubota and T. Yamaguchi, Confocal micro-PIV velocity measurements of physiological fluids in a rectangular microchannel 3rd IASTED ICB_-BioMech (Benidorm, Spain) Proc. 485 (2005).

9. R. Lima, S. Wada, K. Tsubota and T. Yamaguchi, Confocal micro-PIV measurements of three dimensional profiles of cell suspension flow in a square microchannel, Measurement Science and Technology, 17, 797 (2006).

10. R. Lima, T. Ishikawa, S. Tanaka, T. Motohiro, K. Tsubota, S. Wada and T. Yamaguchi, Velocity measurements of blood flow in a rectangular PDMS microchannel assessed by confocal micro-PIV system,WC 2006, Korea, (2006). 11. M. Raffel, C. Willert and J. Kompenhans, Particle image velocimetry: a practical guide. Springer-Verlag Germany, (1998).

12. H. Bruus, Theoretical microfluidics Lecture notes MIC Department of Micro and Nanotechnology Technical University of Denmark Denmark, (2004). 